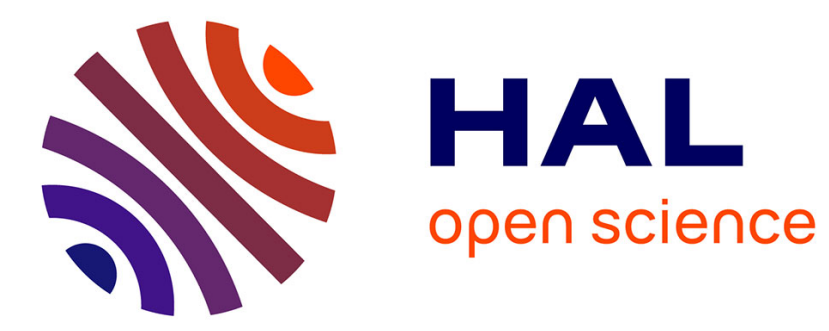

\title{
Developing and Evaluating a Non-Visual Memory Game
} Ravi Kuber, Matthew Tretter, Emma Murphy

\section{To cite this version:}

Ravi Kuber, Matthew Tretter, Emma Murphy. Developing and Evaluating a Non-Visual Memory Game. 13th International Conference on Human-Computer Interaction (INTERACT), Sep 2011, Lisbon, Portugal. pp.541-553, 10.1007/978-3-642-23771-3_41 . hal-01590877

\section{HAL Id: hal-01590877 \\ https://hal.inria.fr/hal-01590877}

Submitted on 20 Sep 2017

HAL is a multi-disciplinary open access archive for the deposit and dissemination of scientific research documents, whether they are published or not. The documents may come from teaching and research institutions in France or abroad, or from public or private research centers.
L'archive ouverte pluridisciplinaire HAL, est destinée au dépôt et à la diffusion de documents scientifiques de niveau recherche, publiés ou non, émanant des établissements d'enseignement et de recherche français ou étrangers, des laboratoires publics ou privés. 


\title{
Developing and Evaluating a Non-Visual Memory Game
}

\author{
Ravi Kuber ${ }^{1}$, Matthew Tretter ${ }^{1}$ and Emma Murphy ${ }^{2}$ \\ ${ }^{1}$ UMBC, 1000 Hilltop Circle, Baltimore MD 21250, USA \\ ${ }^{2}$ Dublin City University, Glasnevin, Dublin 9, Ireland \\ rkuber@umbc.edu
}

\begin{abstract}
This paper describes the development of a non-visual memory game based on the classic game 'Simon ${ }^{\mathrm{TM}}$ ', where users are presented with a sequence of stimuli, which they need to replicate in the same order to progress to the next level. Information is presented using a combination of speech, nonspeech audio and/or haptic cues, designed to aid blind users who are often excluded from mainstream gaming applications. Findings from an empirical study have revealed that when haptic feedback was presented in combination with other modalities, users successfully replicated more sequences, compared with presenting haptic feedback alone. We suggest that when developing a nonvisual game using an unfamiliar input device, speech-based feedback is presented in conjunction with haptic cues.
\end{abstract}

Keywords: Audio, blind, haptics, memory games, multimodal, speech

\section{Introduction}

The recent surge in the availability of brain and memory training games suggests that individuals are interested in keeping their minds active, and strengthening their motor skills through interacting with the software. While these games are typically popular with children and younger adults, research has shown that cognitive training can in certain instances benefit older individuals (Lustig et al., [15]), some of whom may experience levels of memory loss. As much of the information presented via memory game interfaces is visual in nature, individuals who are blind can experience some difficulties accessing content when using their existing assistive technologies. Screen readers, which are often used to convey information from a graphical user interface through speech, inadequately handle graphics, and can omit structural information, making the process of playing a game more complex. In order to provide a more inclusive experience, a need has been identified to replace the missing structure of the interface through the use of alternative channels, such as audio and haptics. 


\section{Related Work}

\subsection{Non-Visual Games}

A variety of games have been developed to address the needs of the blind community. For example, Sanchez [20] used spatialized audio in the design of a twoplayer game (AudioBattleShip) which provides the user with awareness of current position, and informs him/her of key events (e.g. the resulting outcome of dropping a bomb in a cell of the contender's battlefield). Input is made using a tablet, with a pen device aiding the triggering of events. More recently, arcade-style games have been modified specifically for blind users. Examples include an accessible version of 'Rockband' (Allman et al., [1]), where the user is presented with vibrations on the arm and ankle to represent the drum cues, while auditory information is used to provide feedback on performance. Morelli et al. [18] have developed 'VI Bowling', an exergame where blind individuals interact with a wireless controller which provides vibrotactile cues to the user's hand. The user is able to identify the direction of the virtual pins using this feedback. Findings showed that participants were able to throw a virtual ball with an average error of 9.76 degrees, demonstrating the promise of using the 'tactile dowsing' method developed, to aid interaction with a gaming interface.

In terms of memory games, Sjostrom [21] developed a non-visual interface specifically enabling children who are blind, to match pairs of sonified buttons together. Haptic feedback is also produced to provide structural cues to users, presented via the PHANTOM device. Once correctly identified, the pair disappear from the interface leaving a smaller selection of buttons to choose from. Wang et al. [24] developed a similar game using tactile feedback presented via the STRESS ${ }^{2}$ display. Using a range of tactile rendering techniques, the researchers created a set of discernable effects, which the user can explore using his/her sense of touch, to locate matching stimuli. Evreinova et al. [6] developed a memory game targeted to the needs of deaf and hard of hearing users, where participants explore vibrotactile patterns using the Logitech IFeel mouse [14]. The parameters of frequency and duration were modified to develop the set of effects for the game. Interestingly, rather than committing the whole tactile patterns to memory, participants were noted to recall the number of bursts of tactile information associated with each tactile icon. Other strategies were also developed by users to aid game play.

\subsection{Non-Visual Memory}

While research has traditionally focused on human abilities to recognize visual stimuli, less is known about our memory for both haptic and auditory (non-speech) items. In terms of audio, parameters of pitch and tempo of melodies can be effectively recalled by users $[12,13]$. In terms of touch, estimates suggest that we are able to remember between two to six pieces of tactile information (Watkins and 
Watkins, [25]; Mahrer and Miles, [16]). Kuber and Yu [9] found that a sequence of four pin-based tactile icons could be recognized from a wider range presented. Participants were provided with an extensive training period to commit tactile stimuli to memory, which aided retention over the month-long period. However, findings from a follow-up study showed that after a gap of four months without practice of tactile passwords, rates of accurate identification reduced to $58.3 \%$ [10].

\subsection{Non-Visual Design Considerations}

Blind users often rely on keystrokes to navigate around an interface using a screen reader (e.g. up arrow to move up and down arrow to move down), rather than using a mouse. Interacting with a mouse requires a strong level of hand-eye coordination, so when developing a non-visual interface using such a device, it is essential to provide additional non-visual support to aid the targeting process. Furthermore, additional assistance is needed to remain positioned upon targets after locating them, as unintentional deviations may be made. Oakley et al. [19] found that attraction effects (gravity wells) and recess effects were the most effective methods to aid the targeting process, as it was difficult to slip away from a particular object mapped to these properties. Vitense et al. [22] highlighted the benefits that force-feedback cues can make when presented alone and in combination with other forms of feedback, when exploring an interface. The researchers have suggested that conditions providing haptic effects were more quickly recognized than conditions that did not provide haptic feedback.

To ensure that abstract auditory cues (earcons) can be appropriately perceived when integrated with an interface, Brewster et al. [2] have suggested maintaining the level of pitch no higher than $5 \mathrm{Khz}$ and no lower than $125-150 \mathrm{~Hz}$. In addition, manipulating the spatial location of the earcon can be beneficial to distinguish between effects. Findings from a later study revealed that earcons can be recalled over longer periods of time, although training techniques were found to affect recall rates (Brewster, [3]). Studies have shown that auditory feedback can be used to augment haptic cues, due to the mechanical limitations associated with force-feedback devices (McGee et al., [17]) However, research has yet to focus on the memorability of multimodal cues within memory games.

The research described in this paper, examines how effectively sequences of nonvisual effects can be replicated, with the long term goal of developing design guidance for interface developers interested in improving access to memory games, through the use of non-visual feedback. More specifically, we have aimed to determine whether haptic feedback can be recalled more effectively if presented independently or in conjunction with other forms of feedback. 


\section{Development of Memory Game}

'Simon ${ }^{\mathrm{TM}}$ ' [7] is an electronic game, where the user is presented with a sequence of flashing colored lights and tones from an electronic device. The user is required to replicate the sequence, by pressing the colored buttons on the device in exactly the same order as originally presented. A multimodal game has been developed based on Simon $^{\mathrm{TM}}$ (Figure 1). This was designed with the aim of being accessible to users who are blind. The user is presented with the following forms of feedback: speech, nonspeech audio, haptics, and graphics. The user can use one or more forms of each type of feedback to play the game.

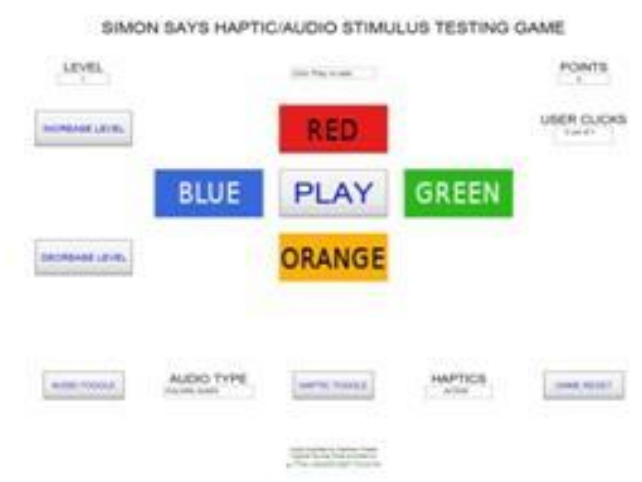

Fig. 1. Accessible multimodal memory game

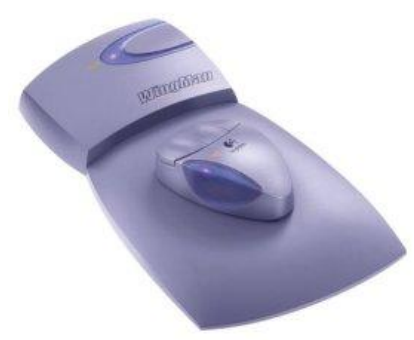

Fig. 2. Logitech Wingman force-feedback mouse [14]

Blind users have the choice of using the keyboard, or using both the keyboard and the Logitech Wingman force-feedback mouse (Figure 2) to interact with the game. The interface contains four different-colored buttons (labeled 'red,' 'blue,' 'green' or 'orange') arranged in the shape of a cross. Each button is mapped to an earcon or speech icon, which is presented for a period of $100 \mathrm{~ms}$ at $60 \mathrm{~dB}$ using recommendations from [5] (Table 1). The buttons were recreated in the auditory domain using non-speech sound by manipulating the pitch and spatial position of a pure sine tone. Upward movements are indicated by a 1 second sine tone with an upward frequency glissando $(220 \mathrm{~Hz}-880 \mathrm{~Hz})$ and a sine tone with a downward glissando indicates a downward movement $(880 \mathrm{~Hz}-220 \mathrm{~Hz})$. Left and right positions were implemented by adjusting the spatial position of a 1 second sine tone $(440 \mathrm{~Hz})$ earcon accordingly Haptic spring effects were developed and integrated with the interface to provide guidance towards a target (button labeled 'red,' 'blue,' 'green' or 'orange'). For example, to prompt the user to move rightwards, the mouse gently guides the users' hand towards the right-hand side of the page.

To play the game, the user is presented with a sequence of buttons which flash and/or play one or more non-visual effects described in Table 1. To complete the stage, the user must successfully select the buttons in the same order originally presented. The system presents speech-based feedback to the user, to indicate his/her 
completion and whether the attempt was successful or not. Other buttons are presented on the interface to enable the user to customize the forms of feedback which he/she wishes to use, and to manipulate the complexity of the game (Figure 1).

Table 1. Mappings to non-visual stimuli

\begin{tabular}{|l|l|l|l|}
\hline & Audio & Speech & Haptic \\
\hline $\begin{array}{l}\text { Move } \\
\text { Up }\end{array}$ & $\begin{array}{l}\text { Increase in pitch } \\
\text { (upward frequency } \\
\text { glissando) }\end{array}$ & $\begin{array}{l}\text { Continuous directed guidance } \\
\text { (upwards) to target using attraction } \\
\text { effect }\end{array}$ \\
\hline $\begin{array}{l}\text { Move } \\
\text { Down }\end{array}$ & $\begin{array}{l}\text { Decrease in pitch } \\
\text { (downward frequency } \\
\text { glissando) }\end{array}$ & Down & $\begin{array}{l}\text { Continuous directed guidance } \\
\text { (downwards) to target using } \\
\text { attraction effect }\end{array}$ \\
\hline $\begin{array}{l}\text { Move } \\
\text { Left }\end{array}$ & Sine tone panned left & Left & $\begin{array}{l}\text { Continuous directed guidance } \\
\text { (leftwards) to target using } \\
\text { attraction effect }\end{array}$ \\
\hline $\begin{array}{l}\text { Move } \\
\text { Right }\end{array}$ & Sine tone panned right & Right & $\begin{array}{l}\text { Continuous directed guidance } \\
\text { (rightwards) to target using } \\
\text { attraction effect }\end{array}$ \\
\hline
\end{tabular}

\section{$4 \quad$ Main Study}

The study aimed to address the following hypotheses:

- H1. Participants would achieve greater levels of accuracy replicating sequences when haptic cues are presented in composite with audio and/or speech, rather than by itself.

- H2. Participants would be able to navigate faster using a mouse while confirming actions using keystrokes, compared to using the keyboard for both activities.

Due to practical difficulties associated with obtaining a large number of blind participants, sighted participants were included within this exploratory study. Ten sighted participants (aged between 20 and 30), were blindfolded when interacting with the game, to simulate conditions of being blind. Two legally-blind individuals (one congenitally blind, one adventitiously blind, aged between 20 and 25) were also selected to participate. Participants were provided with a ten minute period of training to familiarize them with the non-visual icons developed. Both blind participants were provided with an additional five minutes of training using the mouse, due to their lack of experience with the device.

Participants were then asked to play the memory game, by working through a series of different levels, corresponding from 'easy' (two stimuli) to 'complex' (seven stimuli) which they needed to replicate in order to progress to the next stage. Each condition presented in Table 2, was randomized to reduce the occurrence of an order 
effect. A pilot study had revealed that presenting separate speech and auditory effects in composite could cause confusion. Icons were developed presenting speech with variations in pitch to indicate to move upwards or downwards, or speech panned in a particular direction, to convey to the user to move left or right. Each condition was presented twice, to gain a more comprehensive overview of results. Auditory and speech based cues were delivered through noise-cancelling headphones.

For purposes of the study, participants were asked to 'think-aloud', describing their experience after performing each condition, and suggesting improvements for the feedback presented. At the end of the study, they were asked to rate perceived levels of cognitive workload experienced under each condition using a Likert scale (1-5).

Table 2. Conditions presented to participants

\begin{tabular}{|l|l|l|}
\hline Method of Interaction & Feedback & Abbreviation \\
\hline Mouse with keyboard & Haptics & MH \\
\hline & Haptics and speech & MHS \\
\hline & Audio and haptics & MAH \\
\hline & Audio, haptics and speech & MAHS \\
\hline Keyboard & Speech & KS \\
\hline & Audio & KA \\
\hline & Audio and Speech & KAS \\
\hline
\end{tabular}

To determine the usability of the game, the time taken to complete each level and the number of successful attempts were automatically logged by the software. Participants were also asked to rate the level of enjoyment experienced (1 to 5), and asked to provide any comments on the methods of interaction used (e.g. use of keyboard vs mouse with keyboard).

\section{$5 \quad$ Results}

\subsection{Replication of Non-Visual Sequences}

The percentage of successful attempts to replicate sequences of stimuli, are shown in Figure 3. A repeated measures ANOVA showed that the level of accurate replication of sequences varied by condition $(\mathrm{F}(2.901,31.910)=7.023, \mathrm{p}=0.01$, Greenhouse-Geisser adjusted). While results from Figure 3 suggested that the KS, MHS and MAHS conditions could be replicated most accurately (i.e. conditions employing speech-based feedback), post-hoc analysis (Bonferroni corrected) could only confirm significant differences between the following conditions (MH vs MHS, MH vs MAH, MH vs MAHS, MH vs KS, MAHS vs KA).

Although findings indicated that the number of accurate replications of sequences did not differ widely depending on method of interaction (Keyboard - M: 74.3\%, SD: 25.7\%; Mouse with keyboard - M: $74.5 \%$, SD: $28.2 \%$ ), a significant effect could not be detected using statistical analysis. 


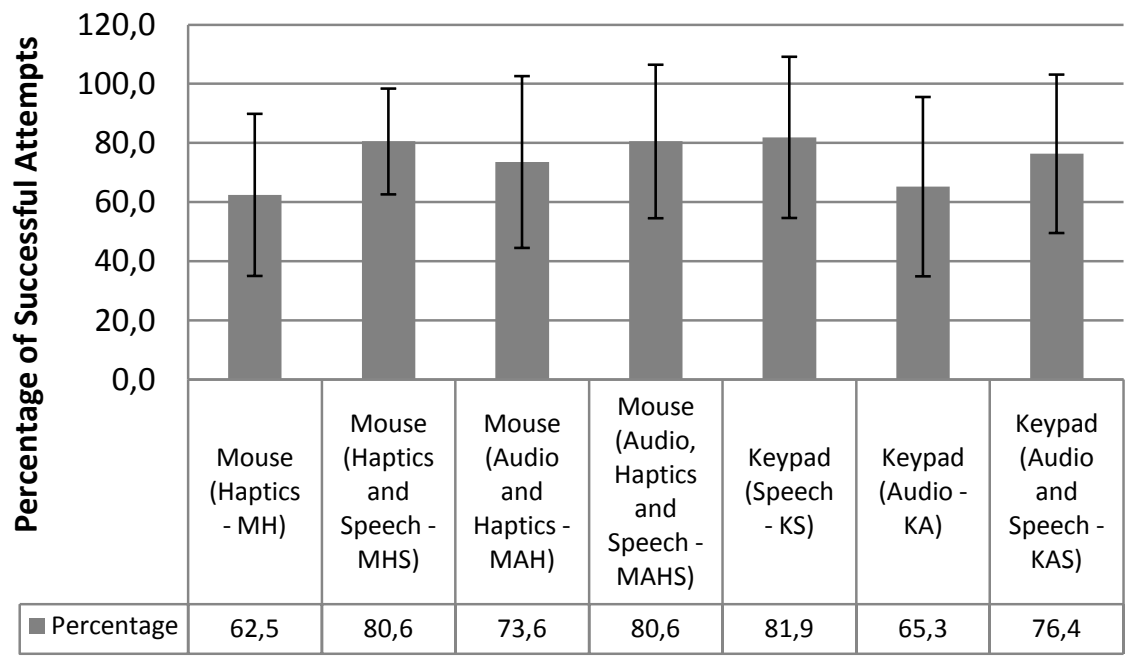

Fig. 3. Percentage of successful attempts by condition

The percentage of successful attempts by level, are shown in Table 3. Results have suggested that as the level of the game increased, accuracy was found to decline from $95.2 \%$ when replicating a sequence of two stimuli to $32.7 \%$ for a sequence of seven stimuli $(\mathrm{F}(4.245,55.181)=72.160, \mathrm{p}=0.00$, Huyhn-Feldt adjusted $)$. The percentage of successful attempts increased upon the second attempt for Levels $3(+1 \%$ difference) and 4 (+9\% difference). However, as the game became more complex resulting in an increase of objects to target, the number of accurate replications on the second attempt appeared to decline.

Table 3. Percentage of successful attempts and time taken by level

\begin{tabular}{|l|l|l|}
\hline Level & $\begin{array}{l}\text { Average Percentage of } \\
\text { Successful Attempts (\%) }\end{array}$ & $\begin{array}{l}\text { Average Time Taken } \\
\text { Seconds) }\end{array}$ \\
\hline 2 & $95.2($ SD: 8.5$)$ & $2.8($ SD: 1.2$)$ \\
\hline 3 & $94.6($ SD: 7.0$)$ & $3.5($ SD: 1.4$)$ \\
\hline 4 & $86.9($ SD: 14.5$)$ & $4.6($ SD: 2.0$)$ \\
\hline 5 & $75.6($ SD: 14.0$)$ & $5.8($ SD: 2.5$)$ \\
\hline 6 & $53.6($ SD: 16.6$)$ & $8.2($ SD: 3.3$)$ \\
\hline 7 & $32.7($ SD: 12.0$)$ & $10.1($ SD: 3.8$)$ \\
\hline
\end{tabular}

\subsection{Time Taken to Perform Tasks}

Figure 4 shows the time spent replicating sequences by condition. A significant effect was detected by condition $(\mathrm{F}(4.034,479.92)=5.227, \mathrm{p}=0.00$, Greenhouse- 
Geisser adjusted $)$ and by level $(\mathrm{F}(3.244,450.929)=294.940, \mathrm{p}=0.00$, GreenhouseGeisser adjusted). Results suggested that participants were on average faster performing tasks under KS, MH and MHS. Post-hoc analysis (Bonferroni corrected) confirmed differences between all levels. However, significant differences were only found between the following conditions: MH vs KA, MHS vs MAH, MHS vs KA, MAH vs KS, KS vs KA.

Although the average time taken for replicating sequences was not found to vary widely between different methods of interaction with the system (Keyboard - M: 5.9 seconds, SD: 3.5 seconds; Mouse with keyboard - M: 5.8, SD: 3.7), further analysis would be needed to confirm the presence of an effect.

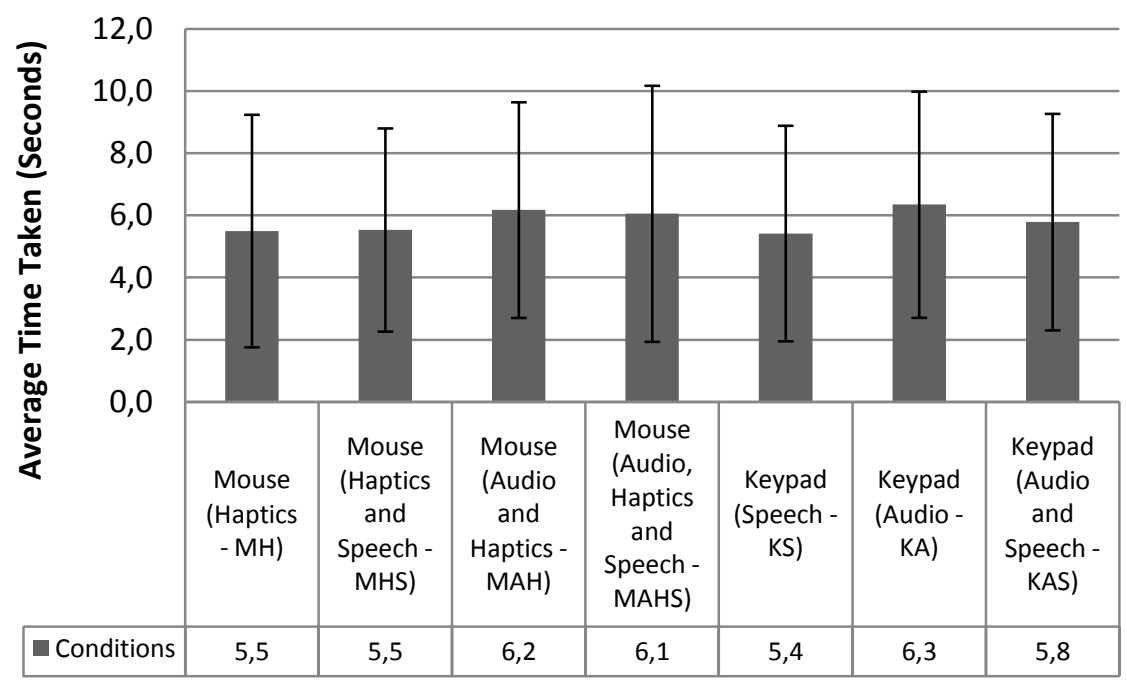

Fig. 4. Time taken to replicate sequences by condition

\subsection{Performance of Blind and Sighted Participants}

Table 4 shows the results of both blind and sighted groups. The two blind participants were on average found to replicate sequences more successfully $(78.0 \%)$ when compared with their sighted counterparts $(73.7 \%)$. Results indicated that blind participants were able to perform tasks within a shorter period of time (4.7s, SD: $2.4 \mathrm{~s}$ ) compared to sighted participants (M: 6.1s, SD: 3.8s), when both using the keyboard or a mouse with keyboard. Table 4 shows that additional time was spent replicating sequences containing non-speech auditory feedback. Results indicated that the time taken was found to increase for both groups while progressing through levels, with a steady decrease in success when replicating sequences of stimuli. However, further study would be needed to identify whether these claims can be validated statistically. 
Table 4. Time taken by condition and level of sight (1-easier to 7-difficult)

\begin{tabular}{|l|l|l|}
\hline Condition & Blind & Sighted \\
\hline MH & 4.4 (SD: 2.1$)$ & 5.8 (SD: 4.0$)$ \\
\hline MHS & 4.5 (SD: 2.3$)$ & 5.8 (SD: 3.4$)$ \\
\hline MAH & 5.0 (SD: 2.5$)$ & 6.5 (SD: 3.6$)$ \\
\hline MAHS & 4.7 (SD: 2.1$)$ & 6.4 (SD: 4.4$)$ \\
\hline KS & 4.2 (SD: 2.7$)$ & 5.7 (SD: 3.6$)$ \\
\hline KA & $5.3($ SD: 2.7$)$ & 6.6 (SD: 3.8$)$ \\
\hline KAS & $4.8($ SD: 2.3$)$ & 6.0 (SD: 3.7$)$ \\
\hline
\end{tabular}

\subsection{Usability of Interface}

Results indicated that levels of workload varied by condition, with larger levels experienced when using the mouse with keyboard, in comparison to solely using the keyboard (Table 5). When recalling the sequence of objects presented, participants reported the most difficulties when using the mouse and keyboard with no audio (MH), and when using the keyboard with auditory feedback (KA). Lower levels of workload were reported for conditions where speech-based feedback was presented (e.g. MHS, KS and KAS). When asked to rate each form of feedback, nine out of the twelve participants agreed with the statement that haptic and speech based cues were appropriate for use. However, only seven agreed with the same statement for audio. Four out of the twelve participants agreed with the statement that using the keyboard with the mouse, was more usable than using the keyboard alone. Nine of the twelve suggested that they were able to enjoy the experience of playing the games developed.

Table 5. Ranking of Ease of Recall and Cognitive Workload by Condition (1-easier/lower to 7difficult/higher)

\begin{tabular}{|l|l|l|}
\hline Condition & Recall & Workload \\
\hline MH & 5.9 (SD: 1.5$)$ & 5.8 (SD: 1.9$)$ \\
\hline MHS & 3.7 (SD: 1.5$)$ & 3.6 (SD: 1.6$)$ \\
\hline MAH & 4.9 (SD: 1.5$)$ & 4.8 (SD: 1.8$)$ \\
\hline MAHS & 3.7 (SD: 2.2$)$ & 4.2 (SD: 1.6$)$ \\
\hline KS & 2.6 (SD: 1.9$)$ & 2.3 (SD: 1.6$)$ \\
\hline KA & 4.5 (SD: 2.2$)$ & 3.9 (SD: 2.1$)$ \\
\hline KAS & 2.7 (SD: 1.3$)$ & 3.3 (SD: 1.4$)$ \\
\hline
\end{tabular}




\section{Discussion}

\subsection{Haptic Feedback vs Multimodal Conditions}

Findings confirmed that participants were able to replicate sequences more accurately when additional feedback was presented alongside haptic feedback (M: 78.2\%, SD: $24.3 \%$ ) compared to the unimodal presentation of the haptic effects (M: $62.5 \%$, SD: $27.4 \%$ ), providing support to H1. Although not statistically significant, results suggested that more time was taken when additional feedback was presented alongside the haptic cues (M: 5.9s, SD: 3.6s), in contrast to haptics presented by itself (M: 5.5s, SD: 3.7s). The large levels of deviation experienced would be worthy of further study. Lower levels of perceived cognitive workload were experienced under conditions where speech was presented (MHS and MAHS), compared with MH and MAH. When questioned about their performance, participants suggested that the presentation of speech would provide the most informative cues which they could commit to memory, while the haptic feedback would provide supplementary feedback to aid retention and provide valuable guidance towards a target.

\subsection{Method of Interaction with Game}

Although findings suggested that the number of accurate replications of sequences and time taken did not differ widely depending on method of interaction, no effects could be detected. Therefore, H2 could not be supported. When questioned about their performance, participants suggested that constraints of the device had slowed their progress. For example, haptic directional effects could be missed, unless the force-feedback mouse was aligned towards the center rather than the edge of its base. Absolute positioning was suggested as one method of improving interaction with the device. Lower levels of cognitive workload were reported for keyboard-only interaction (M: 3.2, SD: 1.7), compared to when using the keyboard in conjunction with the mouse (M: 4.6, SD: 1.3). This was in part attributed to the sensitivity of the mouse, meaning that small movements made using the device, would translate into larger on-screen movements which could cause confusion for the users.

As the blind participants were unfamiliar with using a mouse, it was anticipated sighted users would be faster in terms of targeting objects using this method of interaction. Findings showed that blind participants completed tasks on average 1.4 seconds faster (SD: 1.4 seconds), with greater levels of accuracy (4.3\%) compared with their sighted counterparts. Blind participants were noted to make careful, controlled movements to interact with the device. The blind participants were asked to comment on the condition which most effectively supports mouse use. The combination of speech, audio with haptic feedback (MAHS) was found by both participants to aid interaction with the game. One blind participant mentioned that the "accessibility of the game would enable her to take turns with her sighted siblings, and not be at a disadvantage due to her visual abilities." This is particularly 
encouraging as research suggests that there are few immersive and collaborative games for blind and sighted users (Sanchez, [20]).

\subsection{Auditory and Multimodal Feedback}

Findings from our study revealed that more time was spent using a combination of audio and haptic cues (MAH - M: $6.2 \mathrm{~s}, \mathrm{SD}: 3.5 \mathrm{~s}$ ) compared to the majority of other conditions presented in Figure 4. Cockburn and Brewster [4] found that audio and tactile cues presented individually improved targeting times by $4.2 \%$ and $3.5 \%$, while the combination of audio and tactile feedback reduced normal targeting times by only $1.7 \%$. The researchers have suggested that by combining tactile feedback with 'stickiness' (adjustment of control-display gain), could significantly benefit the targeting process, rather than using alternative combinations of feedback. Subjective comments received from participants in our study, suggested that they experienced difficulties processing both sets of cues in tandem, accounting for the larger levels of cognitive workload expended (M: 4.8, SD: 1.8). More specifically, participants had suggested that the pitch mappings used could on occasion cause confusion, requiring them to think more carefully about the direction of movement which they were meant to convey. Walker and Kramer [23] highlighted that mental models and experiences from previous metaphors can influence a user's perception of pitch movement and associated mappings. This may explain why some users did not intuitively associate the upwards or downwards glissando with the intended movements. In future versions of the system, the auditory and haptic feedback will be more carefully designed to complement one another, with the aim of reducing the levels of cognitive workload experienced.

\subsection{Effects of Memory}

Although we did not record the stimuli selected by participants when a sequence was erroneously entered, participants were questioned on the most memorable parts of each sequence. All twelve participants strongly agreed with the statement that they found it easier to remember the first few non-visual stimuli, compared to the last few stimuli in each of the longer sequences. Watkins and Watkins [25] have suggested that with auditory input, a strong primacy effect is obtained when recalling tactile information, together with a shorter recency effect. Participants in our study were observed pausing midway through longer sequences, attempting to recall the remaining stimuli. Participants suggested that for longer sequences, they would on occasion attempt to guess the order of the last few remaining stimuli, in order to complete the level. While participants suggested that the presence of speech aided their retention of sequences, Watkins and Watkins [25] remain cautious about the role which verbal labels play, favoring further study in the area. 


\subsection{Participant Selection}

When evaluating accessible interfaces, researchers often encounter challenges recruiting target users. This is in part attributed to the small size of the blind community, and the variability in the levels of residual sight which may impact the way the interface is used. In response, blindfolded sighted users are recruited for preliminary or exploratory studies. Studies have shown that no major differences were identified in response times between groups of sighted and blind users (Ferres et al., [8]). However, results from the authors' study revealed that blind participants were found to select more commands, compared to their sighted counterparts. As many blind users opt to use the keyboard, rather than a mouse, so may require additional training to use these type of input devices. Although we thought that it may create difficulties for the blind users who were unfamiliar with the mouse, results in Section 5.3 have revealed that their careful controlled movements, enabled blind participants to complete tasks with and without a mouse in a faster time than their sighted counterparts. However, we acknowledge this may not be the same case for all blind users. Furthermore, the mental structural representation of interfaces differs between both sighted and blind groups (Kurniawan et al., [11]). This is largely due to the restricted output from a screen reader, leading individuals who are blind to perceive that objects on an interface are spatially presented along one dimension. While sighted users knew from previous experience with web pages, that new content is predominantly in the center of the page, they would move the mouse cursor towards this point to locate targets. The case was more difficult for blind users, who were observed moving around the page searching for a landmark to orientate their position, and then search for the particular target within the sequence. Speech-based cues were described as being essential by the blind participants, to aiding interaction with an unfamiliar device such as a mouse. Further study will also need to be performed with representative users, to ensure that the system meets the diverse needs of the blind community.

\section{Conclusion and Future Work}

This study has demonstrated the potential of non-visual feedback in the design of accessible memory games. Observations revealed that blind users were able to interact with the Logitech Wingman force-feedback mouse, and complete the games in a comparable time to using the keyboard alone. Findings have shown that blind users would benefit from haptic feedback providing directional information, however, more successful attempts at replicating sequences were found when haptic cues were presented in combination with other effects, particularly speech-based cues. This was thought to offer additional support when using an unfamiliar input device, such as a force-feedback mouse. In the future, we aim to examine whether participants are able to strengthen their auditory and haptic memory abilities through playing the game, 
and to see whether with more distinctive forms of feedback, participants are able to replicate sequences within a shorter period of time.

\section{Acknowledgements}

We thank Dr. Henry H. Emurian (UMBC) for his input to this work.

\section{References}

1. Allman, T., Dhillon, R.K., Landau, M.A., Kurniawan, S.H.: Rock Vibe: Rock Band ${ }^{\circledR}$ Computer Games for People with No or Limited Vision. In: ACM SIGACCESS Conference on Computers and Accessibility (ASSETS '09), pp. 51--58, ACM Press, New York (2009)

2. Brewster, S.A., Wright, P.C., Edwards, A.D.N.: An Evaluation of Earcons for use in Auditory Human-Computer Interfaces. In: INTERACT'93 and CHI'93, pp. 222--227, ACM Press, New York (1993)

3. Brewster, S.A. Navigating Telephone-Based Interfaces with Earcons. In: BCS'97, pp. 39--6, Springer Verlag (1997)

4. Cockburn, A., Brewster, S.A.: Multimodal Feedback for the Acquisition of Small Targets. Ergonomics, 48(9), 1129--1150 (2005)

5. Dangerous Decibels, www.dangerousdecibels.org

6. Evreinova, T.G., Evreinov, G., Raisamo, R.: An Alternative Approach to Strengthening Tactile Memory for Sensory Disabled People. Universal Access in the Information Society, 5 (2), 189--198 (2006)

7. Hasbro, www.hasbro.com

8. Ferres, L., Lindgaard, G., Sumegi, L.: Evaluating a Tool for Improving Accessibility to Charts and Graphs. In: ACM SIGACCESS Conference on Computers and Accessibility (ASSETS '10), pp. 83-90, ACM Press, New York (2010).

9. Kuber, R., Yu, W.: Feasibility Study of Tactile-based Authentication. International Journal of Human-Computer Studies, 68, 158-181 (2010)

10. Kuber, R., Yu, W.: Tactile vs Graphical Authentication. In: Eurohaptics LNCS, vol. 6191, pp. 314-319, Springer, Heidelberg (2010).

11. Kurniawan, S.H., Sutcliffe, A.G., Blenkhorn, P.L.: How Blind Users' Mental Models Affect their Perceived Usability of an Unfamiliar Screen Reader. In: INTERACT'03, pp. 631 - 638, IOS Press, Amsterdam, Netherlands (2003)

12. Levitin, D. J.: Memory for Musical Attributes. In: Levitin, D.J. (ed), Foundations of Cognitive Psychology: Core Readings. MIT Press (2002)

13. Levitin, D. J., Cook, P. R.: Absolute Memory for Musical Tempo: Additional Evidence that Auditory Memory is Absolute. Perception \& Psychophysics, 58, 927--935 (1996)

14. Logitech, www.logitech.com

15. Lustig, C., Shah, P., Seidler, R., Reuter-Lorenz, P.A.: Aging, Training, and the Brain: A Review and Future Directions. Neuropsychology Review, 19 (4), 504--522 (2009) 
16. Mahrer, P., Miles, C.: Memorial and Strategic Determinants of Tactile Recency. Experimental Psychology 25 (3), 630--643 (1999)

17. McGee, M.R., Brewster, S.A., Gray, P.D.: The Effective Combination of Haptic and Auditory Textural Information. In: First International Workshop on Haptic Human Computer Interaction LNCS vol. 2058, pp. 118-126, Springer-Verlag, London, UK (2001)

18. Morelli, T., Foley, J., Folmer, E.: VI-Bowling: A Tactile Spatial Exergame for Individuals with Visual Impairments. In: ACM SIGACCESS Conference on Computers and Accessibility (ASSETS '10), pp.179--186 (2010)

19. Oakley, I., Adams, A., Brewster, S.A., Gray, P.D.: Guidelines for the Design of Haptic Widgets. In: BCS'02, pp.195-212, Springer Verlag (2002)

20. Sanchez, J.H.: AudioBattleShip: Blind Learners Cognition through Sound. In: Fifth International Conference Series on Disability, Virtual Reality \& Associated Technologies (ICDVRAT'04), (2004)

21. Sjostrom, C.: Using Haptics in Computer Interfaces for Blind People. In: Extended Abstracts of CHI'01, pp. 245-246, ACM Press, New York (2001)

22. Vitense, H., Jacko, J.A., Emery, V.K.: Multimodal Feedback: Establishing a Performance Baseline for Improved Access by Individuals with Visual Impairments. In: ACM SIGACCESS Conference on Computers and Accessibility (ASSETS'02), pp. 49-56, ACM Press, New York (2002).

23. Walker, B. N., Kramer, G.: Mappings and Metaphors in Auditory Displays: An Experimental Assessment. ACM Transactions on Applied Perception, 2(4), 407-412 (2005)

24. Wang, Q., Levesque, V., Pasquero, J., Hayward, V.: A Haptic Memory Game using the STRESS Tactile Display. In: Extended Abstracts of CHI'06, pp. 271-274, ACM Press, New York (2006)

25. Watkins, M.J., Watkins, O.C.: A Tactile Suffix Effect. Memory and Cognition 5, 529--534 (1974) 